Abstract E-054 Table 2 Correlation between Thrombus density and recanalization success or etiology in MCA only

\begin{tabular}{llll}
\hline (mean, SD) & $\begin{array}{l}\text { Successful recanalization } \\
(\mathbf{n}=\mathbf{5 1})\end{array}$ & $\begin{array}{l}\text { Unsuccessful } \\
\text { recanalization }(\mathbf{n}=14)\end{array}$ & $\begin{array}{l}\mathbf{p} \\
\text { value }\end{array}$ \\
\hline $\begin{array}{l}\text { Mean Clot Density } \\
\text { (HU) }\end{array}$ & $49.2(5.8)$ & $53.5(17.1)$ & 0.37 \\
$\begin{array}{l}\text { Max Clot Density } \\
\text { (HU) }\end{array}$ & $57.4(6.9)$ & $62.1(22.6)$ & 0.46 \\
Mean Ratio & $1.27(0.20)$ & $1.37(0.46)$ & \\
Max Ratio & $1.30(0.19)$ & $1.41(0.59)$ & 0.46 \\
& Large Artery $(\mathbf{n}=10)$ & Cardioembolic $(\mathbf{n}=36)$ & 0.48 \\
Mean Clot Density & $49.8(5.6)$ & $48.8(6.4)$ & 0.62 \\
(HU) & & & 0.44 \\
Max Clot Density & $59.5(8.6)$ & $57.2(6.5)$ & \\
(HU) & & $1.26(0.22)$ & 0.36 \\
Mean Ratio & $1.31(0.15)$ & $1.30(0.21)$ & 0.98 \\
Max Ratio & $1.30(0.17)$ & & \\
\hline
\end{tabular}

Disclosures M. Jagani: None. W. Brinjikji: None. D. Kallmes: 1; C; ev3, MicroVention, Sequent, Codman. 2; C; ev3, Medtronic, Codman. 3; C; Microvention.

\section{E-055 ENDOVASCULAR THERAPY FOR PATIENTS WITH ACUTE LARGE MCA TERRITORY ISCHEMIA: ITS EFFECT ON THE RISK OF SUBSEQUENT DEVELOPMENT OF DEEP VENOUS THROMBOSIS}

Z Li, M Cox. Radiology, Thomas Jefferson University Hospital, Philadelphia, PA

\subsection{6/neurintsurg-2016-012589.127}

Introduction Deep venous thrombosis (DVT) is a well-known complication for patients after acute ischemic stroke, due to decreased mobility of the affected extremities and/or prolonged bedrest. Endovascular recanalization therapy (ERT) for acute ischemic stroke has been shown to be superior to the use of intravenous thrombolytic agents alone. Recent randomized trials demonstrated that qualified patients who presented with acute ischemic stroke and underwent endovascular thrombectomy had improved functional outcome. The authors hypothesize that successful ERT decreases the risk of subsequent DVT development in patients with acute large middle cerebral artery (MCA) territory ischemia.

Methods A retrospective review of an imaging database from January 2011 to January 2016 was performed for patients who had acute large MCA territory ischemia based on CT perfusion and CT angiography and subsequent upper or lower extremity duplex ultrasound and/or chest CT for evaluation of DVT or PE. Patents who underwent successful ERT were identified based on operative reports from electronic medical record. Ultrasound or CT reports were retrieved from PACS to identify patients who developed subsequent DVT or PE. Statistical analysis was performed using IBM SPSS 21.0.

Results A total of 88 patients with acute large MCA territory ischemia secondary to major vessel occlusion were identified. Sixty-three patients, 26 (41.3\%) male and 37 (58.7\%) female, either were not qualified for ERT or had persistent occlusion despite attempted ERT. The remaining 25 patients, 11 (44.0\%) male and 14 (56.0\%) female, had complete revascularization (TICI 3). Number of patients who received intravenous tPA was $23(36.5 \%)$ in the occluded group and $11(44.0 \%)$ in the revascularized group, respectively. The percentage of patients who developed subsequent DVT or PE was greater in the occluded group than the revascularized group $(14.3 \%$ vs. $4.0 \%)$. However, the difference was not statistically significant $(\mathrm{p}=0.159)$. Interestingly, $80 \%$ of patients developed DVT on the side of the extremity contralateral to the affected cerebral hemisphere or the expected side of the limb affected by the stroke.

Conclusions In patients with acute large MCA territory ischemia, endovascular recanalization therapy appeared to decrease the risk of developing subsequent DVT or PE. However, a larger sample size is needed to demonstrate statistical significance. Interestingly, majority of DVT were developed on the side of the extremity contralateral to the affected cerebral hemisphere.

Disclosures Z. Li: None. M. Cox: None.

\section{E-056 ASSOCIATION OF ENGORGED PERFORATING ARTERY OF BASILAR TOP WITH NON-ANEURYSMAL PERIMESENCEPHALIC SUBARACHNOID HEMORRHAGE}

B Moon, S Park, K Jang, D Jang. Neurosurgery, Incheon St. Mary's Hospital, Incheon, Republic of Korea

\subsection{6/neurintsurg-2016-012589.128}

Introduction Perimesencephalic subarachnoid hemorrhage (PM$\mathrm{SAH}$ ) accounts for about $10 \%$ of all SAHs, and digital subtraction angiography (DSA) is mostly found to be normal in such patients. The purpose of this study is to investigate the relationship between counts and diameter of engorged perforating artery of basilar top and non-aneurysmal PM-SAH.

Methods DSA findings of all patients who underwent catheter angiography for evaluation of non-aneurysmal PM-SAH between May 2014 and March 2015 were reviewed. Patients with anterior circulation aneurysms were excluded. PM-SAH and control group were evaluated by DSA 3D reconstruction images. Perforating artery diameters were measured and were counted engorged artery. Non-aneurysmal PM-SAH were identified: (1) center of bleeding located immediately anterior and in contact with the brain stem in the prepontine, interpeduncular, or posterior suprasellar cistern; (2) blood limited to the prepontine, interpeduncular, suprasellar, crural, ambient, and/ or quadrigeminal cisterns and/or cisterna magna; (3) no extension of blood into Sylvian or interhemispheric fissures; (4) intraventricular blood limited to incomplete filling of the fourth ventricle and occipital horns of the lateral ventricles (ie, consistent with reflux); (5) no intraparenchymal blood.

Results 4 patients with non-aneurysmal PM-SAH and control group with posterior circulation aneurysms or dissection were identified. In patients with non-aneurysmal PM-SAH, mean diameters and counts of perforating artery were $1.002 \mathrm{~mm}$ (min-max, 0.85-1.26) and 3.75 (min-max, 3-4). In control group, diameters and counts were $0.663 \mathrm{~mm}$ (min-max, 0.51.12) and 2.4 (min-max, 1-4).

Conclusions There is a relationship between PM-SAH and engorged perforating artery counts and diameters. In patients of PM-SAH, there were found increased counts and diameters of perforating artery of basilar top. 


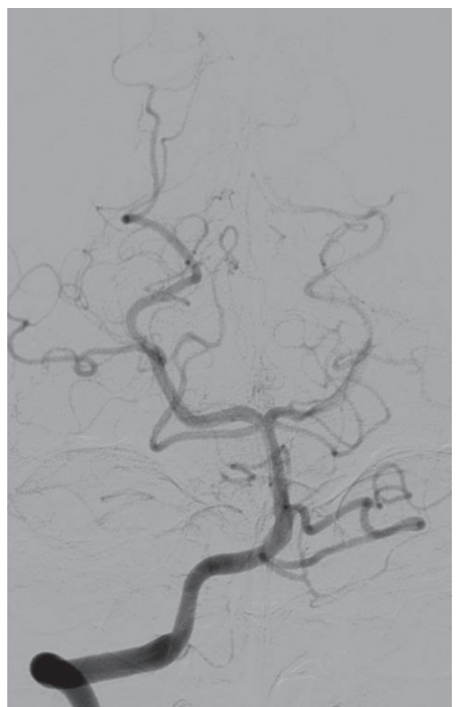

Abstract E-056 Figure 1

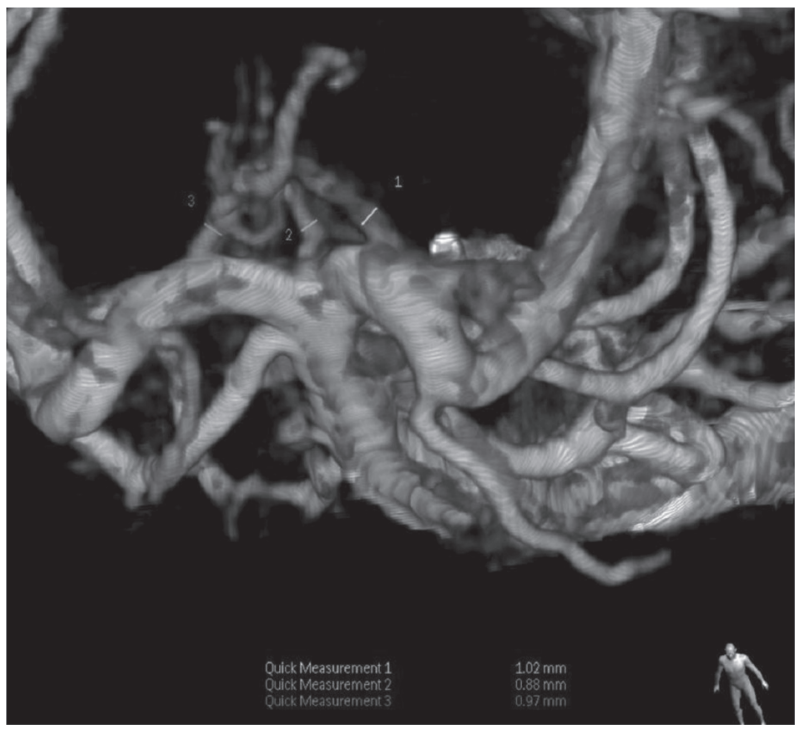

Abstract E-056 Figure 2

Disclosures B. Moon: None. S. Park: None. K. Jang: None. D. Jang: None.

\section{E-057 PROCESS IMPROVEMENT IN DOOR-TO-GROIN PUNCTURE TIMES AT A COMPREHENSIVE STROKE CENTER SHOWS A TREND TOWARDS REDUCED MORTALITY AT 90 DAYS}

${ }^{1} \mathrm{Y}$ Kayan, ${ }^{1} \mathrm{~J}$ Delgado Almandoz, ${ }^{2} \mathrm{M}$ Young, ${ }^{1} \mathrm{f}$ Fease, ${ }^{1} \mathrm{~J} S \mathrm{Scholz},{ }^{1} \mathrm{~A}$ Milner, ${ }^{2} \mathrm{~T}$ Hehr, ${ }^{3} \mathrm{M}$ Mulder, ${ }^{2} \mathrm{P}$ Roohani, ${ }^{2} \mathrm{R}$ Tarrel. ${ }^{1}$ Neurointerventional Radiology, Abbott Northwestern Hospital, Minneapolis, MN; ${ }^{2}$ Vascular Neurology, Abbott Northwestern Hospital, Minneapolis, MN; ${ }^{3}$ Critical Care, Abbott Northwestern Hospital, Minneapolis, MN

\subsection{6/neurintsurg-2016-012589.129}

Introduction Safe and effective endovascular treatment of acute ischemic stroke is dependent on prompt intervention. In light of the positive trials published last year, we intensified our efforts to improve the process of taking patients from the emergency department to the angiography suite for mechanical thrombectomy.

Methods At the beginning of 2015, a process improvement project to improve door-to-groin puncture times for mechanical thrombectomy for emergent large vessel occlusions was undertaken at our institution. After a systematic analysis of the process, the following key changes were standardized: early consultation with the neurointerventionalist for patients presenting with a high National Institutes of Health Stroke Scale (NIHSS $\geq 6$ ), the stroke neurologist and the neurointerventionalist meet the patient in the emergency department for transfers or meet in the CT department for non-transfers, elimination of emergency physician triage for medically stable patients, elimination of lab tests such as creatinine, establishment of a minimum level of clinical information required for the sedation nurse to care for stroke patients safely, institution of a "stroke bag" containing all necessary devices to perform efficient thrombectomy, standardized setup of the procedure table and devices for all operators, calling for patients when the sedation nurse is ready without waiting for the entire team, elimination of unnecessary preoperative steps (e.g. Foley catheter placement, groin shaving, checking of distal pulses), and institution of an abbreviated time-out procedure. All attempted thrombectomies performed in 2013 and 2014 were compared to those performed in 2015 visà-vis the following variables: door-to-groin puncture time, symptomatic intracranial hemorrhage $(\mathrm{SICH})$ rate as defined by SITS-MOST criteria, 90 day mortality rate, and 90 day rate of good clinical outcome defined by a modified Rankin Scale (mRS) of 0-2. A one-tailed Student's t-test and Fisher's exact tests were performed. A p-value $<0.05$ was considered statistically significant.

Results From January 2013 to December 2015, we attempted 108 mechanical thrombectomies. Of these, 43 were in 2015 (40\%). Both the mean and median door-to-groin puncture times were significantly reduced from the previous two years (35 minutes versus 89 minutes and 22 minutes versus 73 minutes, respectively, $\mathrm{p}<0.001)$. There was a trend towards reduced mortality at 90 days $(16 \%$ versus $26 \%, \mathrm{p}=0.166)$. The rates of SICH and mRS 0-2 at 90 days were not significantly different (see Table 1).

Conclusion Our systematic process improvement initiative significantly reduced door-to-groin puncture times and showed a trend towards reduced mortality at 90 days. A multidisciplinary approach and ensuring hospital system investment are key to an effective process.

\begin{tabular}{llll}
\multicolumn{4}{l}{ Abstract E-057 Table 1} \\
\hline & 2013 and 2014 & 2015 & p-value \\
\hline Thrombectomies & 65 & 43 & \\
Door-to-groin puncture & 89 & 35 & $<0.001$ \\
Mean time (minutes) & 73 & 22 & \\
Median time (minutes) & & & \\
SICH & $3(5 \%)$ & $3(7 \%)$ & 0.452 \\
90 day mortality & $17(26 \%)$ & $7(16 \%)$ & 0.166 \\
90 day mRS 0-2 & $24(37 \%)$ & $18(42 \%)$ & 0.376 \\
\hline
\end{tabular}

Disclosures Y. Kayan: 2; C; Medtronic, Penumbra. J. Delgado Almandoz: 2; C; Medtronic, Penumbra. M. Young: None. J. Fease: None. J. Scholz: None. A. Milner: None. T. Hehr: None. M. Mulder: None. P. Roohani: None. R. Tarrel: None. 